\title{
Prospective Association of Handgrip Strength with Risk of New-Onset Cognitive Dysfunction in Korean Adults: A 6-Year National Cohort Study
}

\author{
Sanghoon Jeong ${ }^{1}$ and Junghoon Kim ${ }^{2}$ \\ ${ }^{1}$ Department of Marine Sports, Pukyong National University, Busan, Republic of Korea \\ ${ }^{2}$ Department of Preventive Medicine, Gachon University College of Medicine, Incheon, Republic of Korea
}

\begin{abstract}
Dementia is one of the priority public health problems in the older population, and the number of people with dementia is steadily increasing. The longitudinal association of muscle strength with risk of new-onset cognitive dysfunction in a general population including middle and older adults remains unknown. The purpose of this study was to investigate the effects of low muscle strength on risk for new-onset cognitive dysfunction over 6 years using a large nationwide sample of cognitively healthy adults. Study participants included 6,435 middle and older adults (33,554 person-years of follow-up), using data from the Korean Longitudinal Study of Ageing 2006-2012. Muscular strength was measured using the maximum handgrip strength of each participant as an index of muscle quality. Low muscle strength was defined as one standard deviation below the mean using the handgrip strength index based on the study population. Cognitive function was evaluated using the Mini-Mental Status Evaluation. The hazard ratio (HR) for cognitive dysfunction significantly and linearly increased according to muscle strength status independent of potential confounding factors (HR: 1.36, 95\% confidence interval [Cl]: 1.18-1.56 for low vs. normal-high group). Using stratified analyses, a significant association between muscle strength status and risk of cognitive impairment was observed in those with low physical activity, but not those with high physical activity. We show that handgrip strength is associated with increased risk of new-onset cognitive dysfunction over 6 years of follow-up in cognitively healthy middle aged and older adults at baseline.
\end{abstract}

Keywords: cognitive function; cognitive impairment; handgrip strength; muscle strength; sarcopenia Tohoku J. Exp. Med., 2018 February, 244 (2), 83-91. C 2018 Tohoku University Medical Press

\section{Introduction}

Dementia is one of the priority public health problems in the older population. The number of people with dementia is steadily increasing, faster even than its prevalence (Alzheimer's Disease International 2015). The World Alzheimer Report 2015 estimated that 46.8 million people worldwide were living with dementia at that time, and the number of people with dementia was estimated to double every 20 years, reaching 74.7 million by 2030 (Alzheimer's Disease International 2015). Moreover, the estimated annual cost of dementia was US\$818 billion worldwide in 2015 , which was $1.09 \%$ of the global GDP (Alzheimer's Disease International 2015). Cognitive dysfunction is characterized as a deterioration in cognitive function falling somewhere between normal aging and dementia. Previous studies suggest that $10 \%-15 \%$ of older people with cognitive dysfunction will develop dementia each year, compared with just $1 \%-2 \%$ of older people without cognitive dysfunction (Ferri et al. 2005).
Additionally, cognitive dysfunction is associated with physical functional impairment and decreased quality of life, and is linked to early mortality (Murad et al. 2015; Nishiguchi et al. 2015). Therefore, it is a priority public health issue to identify risk factors that might help prevent individuals from developing cognitive dysfunction.

There is growing evidence for decline in muscle mass and/or strength to readily assess functional and clinical health outcome (Janssen et al. 2002; Bouchard et al. 2009; An and Kim 2016). Handgrip strength is widely used to evaluate muscle strength that is used as a measurement for whole-body muscle strength. This is advantageous because it is easily and safely assessed in older people (Roberts et al. 2011). Previous studies in older people have shown that decreased handgrip strength can predict adverse healthrelated events such as falls, disability, frailty, hospitalization costs, and mortality (Sallinen et al. 2010; Xue et al. 2011; Chen et al. 2012; Guerra et al. 2015), and it is commonly used as an objective measure of muscle strength in epidemiological studies.

Received November 15, 2017; revised and accepted January 18, 2018. Published online February 3, 2018; doi: 10.1620/tjem.244.83. Correspondence: Junghoon Kim, Ph.D., Department of Preventive Medicine, Gachon University College of Medicine, 155 Gaetbeol-ro, Yeonsu-gu, Incheon 21999, Republic of Korea. e-mail: junghoonkim@gachon.ac.kr 
Previous studies with cross-sectional designs have reported that decline in muscle mass or strength was associated with cognitive function in the elderly (Anstey and Smith 1999; Nourhashemi et al. 2002; Chang et al. 2016). For example, Nourhashemi et al. (2002) showed that low muscle mass was associated with cognitive impairment in 7,105 older women. Another study reported that handgrip strength can explain differences in cognitive performances, using data from 180 community-dwelling women aged 60-90 years (Anstey and Smith 1999). Moreover, the meta-analysis of seven cross-sectional studies shows that sarcopenia is defined as low muscle mass and/or function (handgrip strength or gait speed) that is independently associated with cognitive impairment in elderly individuals (Chang et al. 2016). Although the association between muscle mass or strength and cognitive function has been well documented cross-sectionally in elderly individuals, longitudinal studies are still required to understand the causality of these associations. Moreover, a recent review of longitudinal studies that have investigated the association between sarcopenia (decline in muscle mass or strength) and cognitive function indicated a decline in elderly individuals (Fritz et al. 2017). However, the prospective association is still not certain in the general population, including in middle-aged adults (Alfaro-Acha et al. 2006; Mori et al. 2016). No studies on the causal relationship between lower muscle strength and new-onset cognitive dysfunction in cognitively healthy middle-aged and older adults are available.

We therefore assessed the longitudinal relationship between muscle strength using handgrip strength as a measure of muscle quality and the risk of new-onset cognitive dysfunction in a general population of Korean middleaged and older adults using data collected from a national cohort study over six years.

\section{Materials and Methods}

\section{Study Population}

Data from the Korean Longitudinal Study of Ageing (KLoSA) were used in the study. The KLoSA is an ongoing nationwide cohort study of a nationally representative sample of Korean adults aged $\geq 45$ years. Details of the study participants, design and methods used in KLoSA are reported in our previous study (Jang and Kim 2015). In brief, the KLoSA aims to obtain fundamental data that can be used to inform and establish social and economic policies in an ageing society (KEIS, Korea Employment Information Service 2015). In 2006, a total of 10,254 participants completed the baseline survey conducted using the Computer Assisted Personal Interviewing method, and were followed-up in a 2-year cycle until 2012. In the present study, to determine the risk of developing cognitive dysfunction, we excluded participants who had cognitive dysfunction (MiniMental Status Evaluation (MMSE) score $<24$ ) or those who had dementia at baseline $(\mathrm{n}=2,955)$ obtained from family interviews. We also excluded 299 participants who had missing data for handgrip strength, and 565 subjects were excluded due to missing data for other variables used as covariates. Therefore, a total of 6,435 participants (3,220 male and 3,215 female) were included. The KLoSA study was approved by the Institutional Review Board of the Korea Employment Information Service (IRB: No-33602) and all participants provided written informed consent. All methods were performed in accordance with relevant guidelines and regulations.

\section{Cognitive function measure}

We assessed cognitive function using the MMSE Korean version score for each participant at baseline and during the follow-up period at each 2-year cycle (Kim et al. 2003). The MMSE is a brief instrument developed to measure global cognitive performance, and it can screen for dementia. The MMSE comprises 11 questions covering five areas of cognitive function such as orientation in time and place, registration of three objects, attention and calculation, memory (recall of three words), and language (Folstein et al. 1975, Kim et al. 2003). We defined normal cognitive function as having an MMSE $\geq 24$ and cognitive dysfunction as an MMSE $<24$ (Jang and Kim 2015).

\section{Muscular strength}

Muscular strength was measured using the maximum handgrip strength of each participant. Handgrip strength was assessed at least twice using a dynamometer $(\mathrm{kg})$, with the participant in a seated or standing position, their elbow by their side and flexed at right angles, and a neutral wrist position. We calculated the mean of the maximum handgrip strength from both hands. We used handgrip strength as an index of muscle quality (Hamer et al. 2015; Cuthbertson et al. 2016). To investigate the effect of muscle strength on cognitive dysfunction risk, we divided the participants into four groups based on sexspecific categories (Janssen et al. 2002; An and Kim 2016): low ( $<$ mean value minus one standard deviation [SD]), normal-low (mean value minus one $\mathrm{SD}$ to $<$ mean value), normal-high (mean value to $<$ mean value plus one SD), and high ( $\geq$ mean value plus one SD). The cut-off points were $<25.0$ (low), 25.0- $<29.0$ (normal-low), 29.0 - $<32.5$ (normal-high), and $\geq 32.5 \mathrm{~kg}$ (high) for male participants; $<14.5$ (low), 14.5- $<17.5$ (normal-low), 17.5- $<20.0$ (normalhigh), and $\geq 20.0 \mathrm{~kg}$ (high) for female, respectively.

\section{Assessment of other variables}

We considered for baseline characteristics as potential confounders. The information of demographics (age, sex, education level, household income, and living status), health-related behaviors (physical activity and smoking status), body mass index (BMI), and clinical health conditions were obtained from personal interview. Household income was classified in terms of quartiles of the overall population. Education level was classified as: $\leq$ Middle school, high school, or $\geq$ College. All participants self-reported the frequency of their physical activities (days/week) and duration (minutes). Total activity times were calculated considering the frequency and duration in minutes/week. Participants were also categorized into two groups based on the total physical activity level: $<150$ or $\geq 150 \mathrm{~min} /$ week based on the current guidelines for Korean adults (The Ministry of Health and Welfare 2013). BMI was calculated from body weight and height (weight $/$ height $^{2}$ ), and split into two groups defined as normal and obese (BMI $<25$ and $\geq 25 \mathrm{~kg} / \mathrm{m}^{2}$ ) based on a reference for Asian (World Health Organization 2000). Self-reported smoking status was categorized as never, former smoker and current smoker. Clinical health conditions were diagnosed based on self-reported physician diagnosis. We also calculated the number of clinical chronic diseases, including: hypertension, diabetes, cardiovascular disease, 
cerebrovascular diseases, and cancer. These were categorized into three groups as no diseases, one disease, or two or more diseases (An and Kim 2016). We also evaluated the index for depression measured using the Center for Epidemiologic Studies Depression Scale (CESD10) that is a widely used tool to screen for depression (Bjorgvinsson et al. 2013).

\section{Statistical analysis}

All data were analyzed using $\mathrm{R}$ ver. 3.4.1 (R Foundation for Statistical Computing, Vienna, Austria) (R Core Team 2017). We considered $P$-values of $<0.05$ as statistically significant. Baseline characteristics were presented as mean $\pm \mathrm{SD}$ or percentages. To compare baseline characteristics between participants with and without new onset cognitive dysfunction during follow-up, we used chisquared tests for categorical variables and t-tests for continuous variables. We also tested statistical significance for participant characteristics according to muscle strength status using chi-squared tests. Cox proportional hazard models were used to predict the risk of newonset cognitive dysfunction according to muscle strength status, and hazard ratio (HR) and $95 \%$ confidence interval (CI) were estimated. To assess the effects of covariates on the impact of muscle strength on the risk of new-onset cognitive dysfunction, we developed four different sequential models. Model 1 adjusted for age and sex. Model 2 also adjusted for education level, living status and household income. Model 3 further adjusted for smoking status and physical activity. Finally, in model 4 , we also adjusted for obesity, clinical health conditions and CES-D10 score. We also considered linearity according to muscle strength status in all Cox proportional hazard models. In addition, we conducted sensitivity analyses stratified by age groups ( $<60$ years vs. $\geq 60$ years), sex (male vs. female), obesity (obese vs. non-obese), smoking status (non-smoker vs. former/current smoker), physical activity ( $<150 \mathrm{vs.} \geq 150 \mathrm{~min} /$ week), and clinical health conditions (none vs. one or more diseases).

\section{Results}

Table 1 shows the participants' general characteristics at baseline according to incidence of cognitive dysfunction over the 6-year follow-up period. In this study, the mean follow-up period was 5.21 years. A total of 2,092 participants had new-onset cognitive dysfunction during the 33,554 person-years of follow-up. The incidence of cognitive dysfunction was significantly higher in participants who were aged $\geq 60$ years, female, less well-educated, had less income, living alone, lower physical activity, never smoker and those who had medical health condition. The mean handgrip strength was $28.7 \pm 8.3 \mathrm{~kg}$ for participants without cognitive dysfunction and $25.8 \pm 8.0 \mathrm{~kg}$ for participants who developed cognitive dysfunction $(\mathrm{P}<0.001)$. In addition, the CES-D10 score was significantly higher in participants had cognitive dysfunction compared with normal group $(\mathrm{P}<0.001)$.

Table 2 shows the characteristics of participants according to muscle strength status. We found that the frequency of age group, obesity, smoking status, physical activity, and medical health condition were significantly different across muscle strength status (Table 2, $\mathrm{P}<0.05$ ). We also found significant differences in the frequency of participants who had new-onset cognitive dysfunction dur- ing the follow-up period (high: $21.6 \%$, normal-high: $25.3 \%$, normal-low: $36.5 \%$, and low: $49.6 \%, \mathrm{P}<0.001$ ).

Table 3 shows the results of Cox proportional hazard models of muscle strength with the HR for cognitive dysfunction by muscle strength status. Muscle strength status was significantly and linearly associated with increased risk for new-onset cognitive dysfunction after adjusting for age and sex (model 1); in addition to education level, household income, and living status (model 2); and also adjusting for physical activity and smoking status (model 3 ). In the fully adjusted model, muscle strength status showed a significant increased HR for new-onset cognitive dysfunction (HR: 1.36, $95 \%$ CI: $1.18-1.56$ for low group, HR: $1.17,95 \%$ CI: 1.05-1.31 for normal-low vs. normal-high group) after additionally adjusting for obesity, medical health condition and CES-D10 score.

In a sensitivity analysis, the significant association between muscle strength status and risk of cognitive dysfunction was observed in the both middle aged and older adults. Moreover, the significant association between muscle strength status and risk of cognitive dysfunction was observed for those with low physical activity, but not those with high physical activity (Fig. 1). We also found a marginally significant interaction between muscle strength and physical activity on the risk of cognitive dysfunction onset $(\mathrm{P}$-interaction $=0.055)$. Significant associations were observed between muscle strength status and increased risk of new-onset cognitive dysfunction in other stratified models, consistent with findings in the overall participants (Fig. $1)$.

In this study, the cognitive function score in participants who had new-onset cognitive dysfunction was significantly lower than in those without cognitive dysfunction (Table 1). These results suggest that the cognitive function at baseline can affect the association between handgrip strength and the incidence of cognitive dysfunction. Therefore, we also analyzed the association between muscle strength status and an increased risk of new-onset cognitive dysfunction after excluding participants with incidences of cognitive dysfunction within the two-year follow-up period $(n=852)$, and found the results were unchanged (Table 4).

\section{Discussion}

In this large longitudinal study, we investigated the effects of handgrip strength on the risk of new-onset cognitive dysfunction in middle-aged and elderly Korean adults. To our knowledge, this is the first population-based prospective study exploring the relationship between handgrip strength and risk of cognitive dysfunction. Our findings suggest that lower muscle strength (i.e., dynapenia/sarcopenia), evaluated by handgrip strength, is significantly associated with an increased risk of new-onset cognitive dysfunction in cognitively healthy middle-aged and older adults over a 6-year follow-up period, independent of chronic disease, physical activity, and other potential confounding factors. Thus, our findings suggest that the skeletal muscle 
Table 1. Baseline characteristics of study participants according to new-onset cognitive dysfunction during follow-up.

\begin{tabular}{|c|c|c|c|c|}
\hline \multirow[b]{2}{*}{ Characteristics } & \multirow[b]{2}{*}{ Overall $(\mathrm{n}=6,435)$} & \multicolumn{2}{|c|}{ Cognitive dysfunction status during follow-up } & \multirow[b]{2}{*}{ P-value } \\
\hline & & $\begin{array}{l}\text { Normal cognitive function } \\
(\mathrm{n}=4,343)\end{array}$ & $\begin{array}{l}\text { Cognitive dysfunction } \\
(\mathrm{n}=2,092)\end{array}$ & \\
\hline \multicolumn{5}{|l|}{ Age group (\%) } \\
\hline $45-59$ years & 3,837 (59.6) & $2,982(68.7)$ & $855(40.9)$ & \multirow[t]{2}{*}{$<0.001$} \\
\hline$\geq 60$ years & $2,598(40.4)$ & $1,361(31.3)$ & $1,237(59.1)$ & \\
\hline \multicolumn{5}{|l|}{$\operatorname{Sex}(\%)$} \\
\hline Male & $3,220(50.0)$ & $2,242(51.6)$ & $978(46.7)$ & \multirow[t]{2}{*}{$<0.001$} \\
\hline Female & $3,215(50.0)$ & $2,101(48.4)$ & $1,114(53.3)$ & \\
\hline \multicolumn{5}{|l|}{ Education (\%) } \\
\hline$\leq$ Middle school & $3,278(50.9)$ & $1,902(43.8)$ & $1,376(65.8)$ & \multirow[t]{3}{*}{$<0.001$} \\
\hline High school & 2,224 (34.6) & $1,694(39.0)$ & $530(25.3)$ & \\
\hline$\geq$ College & $933(14.5)$ & 747 (17.2) & $186(8.9)$ & \\
\hline \multicolumn{5}{|l|}{ Household income (\%) } \\
\hline Low & $1,472(22.9)$ & $836(19.2)$ & $636(30.4)$ & \multirow[t]{4}{*}{$<0.001$} \\
\hline Lower-middle & $1,712(26.6)$ & $1,078(24.8)$ & $634(30.3)$ & \\
\hline Upper-middle & $1,247(19.4)$ & 895 (20.6) & $352(16.8)$ & \\
\hline High & $2,004(31.1)$ & $1,534(35.3)$ & $470(22.5)$ & \\
\hline \multicolumn{5}{|l|}{ Living status (\%) } \\
\hline Living together & $6,068(94.3)$ & 4,151 (95.6) & 1,917 (91.6) & \multirow[t]{2}{*}{$<0.001$} \\
\hline Living alone & $367(5.7)$ & $192(4.4)$ & $175(8.4)$ & \\
\hline \multicolumn{5}{|l|}{ Physical activity (\%) } \\
\hline$<150 \mathrm{~min} /$ week & $4,391(68.2)$ & $2,915(67.1)$ & $1,476(70.6)$ & \multirow[t]{2}{*}{0.006} \\
\hline$\geq 150 \mathrm{~min} /$ week & 2,044 (31.8) & $1,428(32.9)$ & $616(29.4)$ & \\
\hline \multicolumn{5}{|l|}{ Smoking status (\%) } \\
\hline Never & $4,356(67.7)$ & $2,886(66.5)$ & $1,470(70.3)$ & \multirow[t]{3}{*}{$<0.001$} \\
\hline Former smoker & $668(10.4)$ & $448(10.3)$ & $220(10.5)$ & \\
\hline Current smoker & $1,411(21.9)$ & $1,009(23.2)$ & $402(19.2)$ & \\
\hline \multicolumn{5}{|l|}{ Obesity } \\
\hline No & 4,943 (76.8) & $3,362(77.4)$ & $1,581(75.6)$ & \multirow[t]{2}{*}{0.102} \\
\hline Yes & $1,492(23.2)$ & $981(22.6)$ & $511(24.4)$ & \\
\hline \multicolumn{5}{|l|}{ Medical health condition (\%) } \\
\hline None & $4,405(68.5)$ & $3,137(72.2)$ & $1,268(60.6)$ & \multirow[t]{3}{*}{$<0.001$} \\
\hline One disease & $1,524(23.7)$ & $943(21.7)$ & $581(27.8)$ & \\
\hline Two or more diseases & $506(7.9)$ & $263(6.1)$ & $243(11.6)$ & \\
\hline Handgrip strength $(\mathrm{kg}$, mean $\pm \mathrm{SD})$ & $27.8 \pm 8.3$ & $28.7 \pm 8.3$ & $25.8 \pm 8.0$ & $<0.001$ \\
\hline CES-D10 (score, mean \pm SD) & $3.21 \pm 2.74$ & $2.77 \pm 2.55$ & $3.97 \pm 2.89$ & $<0.001$ \\
\hline MMSE (score, mean \pm SD) & $27.4 \pm 1.6$ & $27.6 \pm 1.5$ & $26.9 \pm 1.7$ & $<0.001$ \\
\hline
\end{tabular}

Values are number $(\%)$ or mean \pm SD. We used chi-squared test for categorical variables and t-tests for continuous variables. Medical health condition was defined as any clinical multi-morbidity of hypertension, diabetes, cardiovascular disease, stroke or cancer; CES-D10, Center for Epidemiological Studies Depression; MMSE, mini mental status evaluation.

strength is an independent risk factor of incidence of cognitive dysfunction in general population.

In the present study, the risk of cognitive dysfunction was approximately 1.4 times higher in the lower muscle strength group compared with those with normal-high muscular strength, after adjusting for potential confounding variables. Our findings support the previous cross-sectional studies that suggest a relationship between skeletal muscle mass or strength and decline in cognition (Anstey and Smith 1999; Nourhashemi et al. 2002; Rosano et al. 2005; Chang et al. 2016). However, most of these observational studies considered only cross-sectional associations of muscular strength and specific cognitive function score, therefore limiting conclusions with regard to long-term decline in muscle strength (Chang et al. 2016). Several studies have investigated the longitudinal association between muscle strength and cognitive function (Fritz et al. 2017). One community-based 3.5-year follow-up study, reported that handgrip strength was linked to the greater variability of memory decline in a small sample of 426 elderly individuals (Christensen et al. 1999), with an alternative study reporting that low handgrip strength was associated with an 
Table 2. Characteristics of study participants according to muscle strength status at baseline.

\begin{tabular}{|c|c|c|c|c|c|}
\hline & \multicolumn{4}{|c|}{ Muscle strength status at baseline } & \multirow{2}{*}{ P-value } \\
\hline & High & Normal-high & Normal-low & Low & \\
\hline \multicolumn{6}{|l|}{ Age groups (\%) } \\
\hline$<60$ years & $799(87.3)$ & $1,597(73.2)$ & $1,195(50.0)$ & $246(25.9)$ & $<0.001$ \\
\hline$\geq 60$ years & $116(12.7)$ & $586(26.8)$ & $1,194(50.0)$ & $702(74.1)$ & \\
\hline \multicolumn{6}{|l|}{$\operatorname{Sex}(\%)$} \\
\hline Male & $475(51.9)$ & $1,099(50.3)$ & $1,164(48.7)$ & $482(50.8)$ & 0.352 \\
\hline Female & $440(48.1)$ & $1,084(49.7)$ & $1,225(51.3)$ & $466(49.2)$ & \\
\hline \multicolumn{6}{|l|}{ Obesity } \\
\hline No & $608(66.4)$ & $1,683(77.1)$ & $1,898(79.4)$ & $754(79.5)$ & $<0.001$ \\
\hline Yes & $307(33.6)$ & $500(22.9)$ & $491(20.6)$ & $194(20.5)$ & \\
\hline \multicolumn{6}{|l|}{ Smoking status $(\%)$} \\
\hline Never & $614(67.1)$ & $1,494(68.4)$ & $1,626(68.1)$ & $622(65.6)$ & 0.011 \\
\hline Former smoker & $93(10.2)$ & $189(8.7)$ & $263(11.0)$ & $123(13.0)$ & \\
\hline Current smoker & $208(22.7)$ & $500(22.9)$ & $500(20.9)$ & $203(21.4)$ & \\
\hline \multicolumn{6}{|l|}{ Physical activity (\%) } \\
\hline$<150 \mathrm{~min} /$ week & $597(65.2)$ & $1,465(67.1)$ & $1,637(68.5)$ & $692(73.0)$ & $<0.001$ \\
\hline$\geq 150 \mathrm{~min} /$ week & $318(34.8)$ & $718(32.9)$ & $752(31.5)$ & $256(27.0)$ & \\
\hline \multicolumn{6}{|l|}{$\begin{array}{l}\text { Medical health condition } \\
(\%)\end{array}$} \\
\hline None & $708(77.4)$ & $1,633(74.8)$ & $1,559(65.3)$ & $505(53.3)$ & $<0.001$ \\
\hline One disease & $171(18.7)$ & $444(20.3)$ & $621(26.0)$ & $288(30.4)$ & \\
\hline Two or more diseases & $36(3.9)$ & $106(4.9)$ & $209(8.7)$ & $155(16.4)$ & \\
\hline \multicolumn{6}{|c|}{ Onset cognitive dysfunction during follow-up (\%) } \\
\hline No & $717(78.4)$ & $1,630(74.7)$ & $1,518(63.5)$ & $478(50.4)$ & $<0.001$ \\
\hline Yes & $198(21.6)$ & $553(25.3)$ & $871(36.5)$ & $470(49.6)$ & \\
\hline
\end{tabular}

Values represent number of occurrences (\%). Analyses used chi-squared tests for categorical variables. Medical health condition was defined as any clinical multi-morbidity of hypertension, diabetes, cardiovascular disease, stroke or cancer.

Table 3. Hazard ratio $(95 \% \mathrm{CI})$ for the new-onset cognitive dysfunction by muscle strength.

\begin{tabular}{|c|c|c|c|c|c|c|c|}
\hline \multirow{2}{*}{ Muscle strength status } & \multirow{2}{*}{$\begin{array}{l}\text { Total person- } \\
\text { years }\end{array}$} & \multirow{2}{*}{$\begin{array}{c}\text { No. of cognitive } \\
\text { dysfunction/participants }\end{array}$} & \multirow{2}{*}{$\begin{array}{l}\text { Event rate (1000- } \\
\text { person year) }\end{array}$} & \multicolumn{4}{|c|}{ HR $(95 \% \mathrm{CI})$} \\
\hline & & & & Model 1 & Model 2 & Model 3 & Model 4 \\
\hline High & 5,054 & $198 / 915$ & 39.2 & $0.84(0.71-0.99)^{*}$ & $0.94(0.80-1.11)$ & $0.94(0.80-1.11)$ & $0.95(0.80-1.12)$ \\
\hline Normal-high & 11,804 & $553 / 2,183$ & 46.9 & 1.00 (Reference) & 1.00 (Reference) & 1.00 (Reference) & 1.00 (Reference) \\
\hline Normal-low & 12,254 & $871 / 2,389$ & 71.1 & $1.51(1.36-1.68)^{*}$ & $1.21(1.08-1.35)^{*}$ & $1.21(1.08-1.35)^{*}$ & $1.17(1.05-1.31)^{*}$ \\
\hline Low & 4,442 & $470 / 948$ & 105.8 & $2.23(1.97-2.52)^{*}$ & $1.38(1.21-1.58)^{*}$ & $1.38(1.21-1.58)^{*}$ & $1.36(1.18-1.56)^{*}$ \\
\hline P-trend & & & & $<0.001$ & $<0.001$ & $<0.001$ & $<0.001$ \\
\hline
\end{tabular}

Values denote hazard ratio $(95 \% \mathrm{CI})$. Model 1 was adjusted for age and sex. Model 2 also adjusted for education, household income, and living status. Model 3 further adjusted for physical activity and smoking status. Model 4 also adjusted for obesity, medical health condition, and CES-D10 score.

CI, confidence interval; CES-D10, Center for Epidemiological Studies Depression.

$*$ P-value $<0.05$ compared with reference group.

increased risk of Alzheimer's disease in cognitively healthy elderly individuals (Boyle et al. 2009). Furthermore, longitudinal studies, based on 20-year follow up data for a population (Sternang et al. 2016), identified that changes in handgrip strength are associated with changes in cognitive abilities. Moreover, a recent review of longitudinal studies investigated the association between sarcopenia (muscle mass and/or strength) and cognitive function, and indicated a decline of these conditions in elderly individuals (Fritz et al. 2017). However, evidence on the contributing relation- 


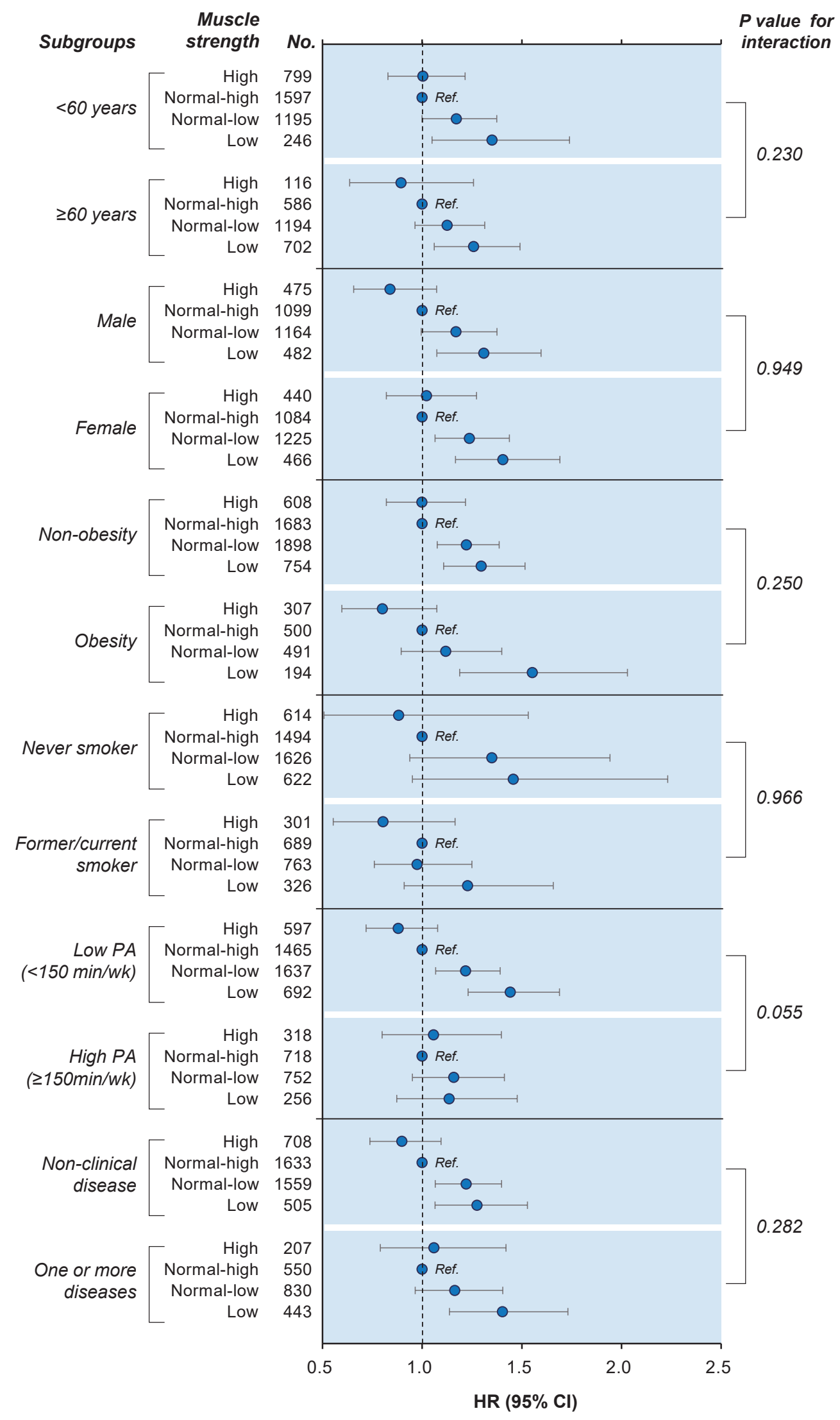

Fig. 1. The risk of cognitive dysfunction according to handgrip strength by subgroups.

All models were adjusted for age, sex, education level, household income, living status, physical activity, smoking status, obesity, clinical health conditions and CES-D10 score. 
Table 4. Hazard ratio $(95 \% \mathrm{CI})$ for the new-onset cognitive dysfunction by muscle strength after excluding participants had an incidence of cognitive dysfunction within two years.

\begin{tabular}{lcc}
\hline Muscle strength status & No. of cognitive dysfunction/participants & HR $(95 \% \mathrm{CI})$ \\
\hline High & $133 / 850$ & $0.95(0.78-1.01)$ \\
Normal-high & $346 / 1,976$ & 1.00 (Reference) \\
Normal-low & $516 / 2,034$ & $1.24(1.08-1.43)^{*}$ \\
Low & $245 / 723$ & $1.39(1.17-1.67)^{*}$ \\
P-trend & & $<0.001$ \\
\hline
\end{tabular}

Values denote hazard ratio (95\% CI). Adjusted for age, sex, education, household income, living status, physical activity, smoking status, obesity, medical health condition, and CES-D10 score.

CI, confidence interval; CES-D10, Center for Epidemiological Studies Depression.

$* \mathrm{P}$-value $<0.05$ compared with reference group.

ship between lower muscle strength and new-onset cognitive dysfunction in the cognitively healthy and the general population are rare, as the majority of previous studies have investigated the older population only. Our findings suggest that muscle strength directly contributes to the future development of cognitive dysfunction, implying that this is an important public health issue that would benefit from improvement with middle-aged adults also being included.

Several biological factors could explain our findings on the longitudinal association between muscle strength and risk of new-onset cognitive dysfunction. One possible explanation for the association between muscle strength and cognitive dysfunction risk pertains to oxidative stress and inflammation (Chi et al. 2017). Oxidative stress and inflammation are well known to be directly linked to a decline in cognitive function (Glade 2010), and may play a role in the onset of cognitive dysfunction (Pedersen and Febbraio 2012). It is understood that skeletal muscle is the target of numerous hormones, and recent evidence has shown that skeletal muscle has a role as a secretory organ of cytokines and other peptides, as well as denominated myokines such as brain-derived neurotrophic factor (BDNF), interleukin-6 (IL-6), IL-8, IL-15, and leukaemia inhibitory factor, which have autocrine, paracrine, or endocrine actions and are involved in inflammatory processes (Pedersen and Febbraio 2012). Decline in muscle mass and strength also may reduce expression of BDNF, insulin-like growth factor-1, both of which are thought to play a role in learning and neural plasticity (Pedersen and Febbraio 2012).

Lower muscle strength that occurs in concert with biological aging may also predict chronic diseases and decline in physical and cognitive function (Bohannon 2008). Higher levels of skeletal muscle mass and strength are associated with a lower risk of frailty, cardiovascular disease, and cardiovascular and all-cause mortality (Janssen et al. 2002; Bouchard et al. 2009; Guadalupe-Grau et al. 2015). Additionally, many studies have shown that physical functional decline and chronic disease are linked with cognitive health decline (Rosano et al. 2005; Auyeung et al. 2008; Nishiguchi et al. 2015). Critically, decline of handgrip strength may represent an age-related change in physical function and progression to frailty, whilst contributing to cognitive decline and increasing the risk of cognitive dysfunction. Taken together, these results provide a possible interpretation of our finding of an association between muscle strength and cognitive dysfunction risk. Additionally, declining central nervous system function with age may be an important mechanism in the association between decline in muscle function and cognitive dysfunction (Baltes and Lindenberger 1997). For example, Salthouse (1996) reported that slow reaction times are associated with cognitive function in the elderly. Moreover, muscle strength may be a general predictor of central nervous system integrity (Salthouse et al. 1998). Thus, muscle strength could be an early marker of a decrease in nervous system function associated with aging, which is reflected in cognitive function (Raji et al. 2005).

Physically inactivity is one of the major factors known to accelerate both muscle loss and strength with aging (Mijnarends et al. 2016). However, functional decline caused by decline in muscle strength also contributes to lack of physical activity. Many previous studies have shown that physical inactivity contributes to cognitive function decline. Furthermore, physically active lifestyles have a benefit in maintaining skeletal muscle mass and strength that may itself contribute to cognitive decline and increasing the risk of cognitive dysfunction. Indeed, we found that the association between muscle strength and the risk of cognitive dysfunction was modified by physical activity status, although the associations were not different across sex, age groups, and other demographic variables. In the present study, the HR for cognitive dysfunction was 1.44-fold higher in participants with low muscle strength than in those with normal-high muscle strength in a low physical activity group, but we did not observe the same association in those with higher levels of physical activity (HR: 1.13 in low group vs. normal-high group, Fig. 1). Several observational and intervention studies have also reported that physically active lifestyles have a benefit in maintaining of cognitive function (Espeland et al. 2017; Jeong and Jang 2017; Zhu et al. 2017). Our findings and 
those of previous studies suggest that participation in physical activity might offset the negative impacts of low muscle strength on risk of cognitive dysfunction.

The major strengths of our study are that it used a prospective design with a 6-year follow-up period in a large, representative sample of adults in the general population. We also controlled for important potential confounding factors including demographics, health-related behaviors, and other clinical health conditions, and index for depression. Nevertheless, some limitations of the present study should be considered. First, we assessed muscle strength by using handgrip strength as a measurement of quality of muscular strength, but did not evaluate muscle mass or other muscle functional parameters that have been shown to predict cognitive decline previously (Auyeung et al. 2008). However, handgrip strength as a measurement of muscle strength and quality is easily and safely investigated, particularly in elderly populations. It is also used as a representative measure of whole-body muscular strength, and it may be an effective index to predict development of cognitive dysfunction. Furthermore, a recent study has shown clearer associations between sarcopenia assessed by muscular strength with health outcomes, then by sarcopenia assessed by muscle mass (Menant et al. 2017). We evaluated cognitive function using only the MMSE scores. The MMSE is an adequate tool for screening dementia in elderly individuals with minimum literacy skills (Scazufca et al. 2009). However, misclassification is also highly unacceptable for elderly individuals who are illiterate (Scazufca et al. 2009).

In conclusion, during a 6-year follow-up period, we found that handgrip strength was strongly associated with an increased risk of new-onset cognitive dysfunction in cognitively healthy middle-aged and older adults at baseline. Furthermore, physical activity may offset the negative impacts of lower muscle strength on the risk of cognitive dysfunction.

\section{Acknowledgments}

This work was supported by the Basic Science Research Program through the National Research Foundation of Korea funded by the Korea Ministry of Education (Grant No. 2014R1A1A2059106, 2017R1D1A1B03035192).

\section{Conflict of Interest}

The authors declare no conflict of interest.

\section{References}

Alfaro-Acha, A., Al Snih, S., Raji, M.A., Kuo, Y.F., Markides, K.S. \& Ottenbacher, K.J. (2006) Handgrip strength and cognitive decline in older Mexican Americans. J . Gerontol. A Biol. Sci. Med. Sci., 61, 859-865.

Alzheimer's Disease International (2015) World Alzheimer Report 2015: The Global Impact of Dementia. https://www.alz.co.uk/research/WorldAlzheimerReport2015.pdf. [Accessed: December 6, 2016].

An, K.O. \& Kim, J. (2016) Association of sarcopenia and obesity with multimorbidity in Korean adults: a nationwide cross- sectional study. J. Am. Med. Dir. Assoc., 17, 960.e1-960.e7.

Anstey, K.J. \& Smith, G.A. (1999) Interrelationships among biological markers of aging, health, activity, acculturation, and cognitive performance in late adulthood. Psychol. Aging, 14, 605-618.

Auyeung, T.W., Kwok, T., Lee, J., Leung, P.C., Leung, J. \& Woo, J. (2008) Functional decline in cognitive impairment: the relationship between physical and cognitive function. Neuroepidemiology, 31, 167-173.

Baltes, P.B. \& Lindenberger, U. (1997) Emergence of a powerful connection between sensory and cognitive functions across the adult life span: a new window to the study of cognitive aging? Psychol. Aging, 12, 12-21.

Bjorgvinsson, T., Kertz, S.J., Bigda-Peyton, J.S., McCoy, K.L. \& Aderka, I.M. (2013) Psychometric properties of the CES-D-10 in a psychiatric sample. Assessment, 20, 429-436.

Bohannon, R.W. (2008) Hand-grip dynamometry predicts future outcomes in aging adults. J. Geriatr. Phys. Ther., 31, 3-10.

Bouchard, D.R., Dionne, I.J. \& Brochu, M. (2009) Sarcopenic/ obesity and physical capacity in older men and women: data from the Nutrition as a Determinant of Successful Aging (NuAge)-the Quebec longitudinal Study. Obesity, 17, 20822088.

Boyle, P.A., Buchman, A.S., Wilson, R.S., Leurgans, S.E. \& Bennett, D.A. (2009) Association of muscle strength with the risk of Alzheimer disease and the rate of cognitive decline in community-dwelling older persons. Arch. Neurol., 66, 13391344.

Chang, K.V., Hsu, T.H., Wu, W.T., Huang, K.C. \& Han, D.S. (2016) Association between sarcopenia and cognitive impairment: a systematic review and meta-analysis. J. Am. Med. Dir. Assoc., 17, 1164.e7-1164.e15.

Chen, P.J., Lin, M.H., Peng, L.N., Liu, C.L., Chang, C.W., Lin, Y.T. \& Chen, L.K. (2012) Predicting cause-specific mortality of older men living in the veterans home by handgrip strength and walking speed: a 3-year, prospective cohort study in Taiwan. J. Am. Med. Dir. Assoc., 13, 517-521.

Chi, G.C., Fitzpatrick, A.L., Sharma, M., Jenny, N.S., Lopez, O.L. \& DeKosky, S.T. (2017) Inflammatory biomarkers predict domain-specific cognitive decline in older adults. J. Gerontol. A Biol. Sci. Med. Sci., 72, 796-803.

Christensen, H., Mackinnon, A.J., Korten, A.E., Jorm, A.F., Henderson, A.S., Jacomb, P. \& Rodgers, B. (1999) An analysis of diversity in the cognitive performance of elderly community dwellers: individual differences in change scores as a function of age. Psychol. Aging, 14, 365-379.

Cuthbertson, D.J., Bell, J.A., Ng, S.Y., Kemp, G.J., Kivimaki, M. \& Hamer, M. (2016) Dynapenic obesity and the risk of incident Type 2 diabetes: the English Longitudinal Study of Ageing. Diabet. Med., 33, 1052-1059.

Espeland, M.A., Lipska, K., Miller, M.E., Rushing, J., Cohen, R.A., Verghese, J., McDermott, M.M., King, A.C., Strotmeyer, E.S., Blair, S.N., Pahor, M., Reid, K., Demons, J. \& Kritchevsky, S.B.; LIFE Study Investigators (2017) Effects of physical activity intervention on physical and cognitive function in sedentary adults with and without diabetes. $J$. Gerontol. A Biol. Sci. Med. Sci., 72, 861-866.

Ferri, C.P., Prince, M., Brayne, C., Brodaty, H., Fratiglioni, L., Ganguli, M., Hall, K., Hasegawa, K., Hendrie, H., Huang, Y., Jorm, A., Mathers, C., Menezes, P.R., Rimmer, E. \& Scazufca, M.; Alzheimer's Disease International (2005) Global prevalence of dementia: a Delphi consensus study. Lancet, 366, 2112-2117.

Folstein, M.F., Folstein, S.E. \& McHugh, P.R. (1975) "Minimental state". A practical method for grading the cognitive state of patients for the clinician. J. Psychiatr. Res., 12, 189-198.

Fritz, N.E., McCarthy, C.J. \& Adamo, D.E. (2017) Handgrip strength as a means of monitoring progression of cognitive 
decline: a scoping review. Ageing Res. Rev., 35, 112-123.

Glade, M.J. (2010) Oxidative stress and cognitive longevity. Nutrition, 26, 595-603.

Guadalupe-Grau, A., Carnicero, J.A., Gomez-Cabello, A., Gutierrez Avila, G., Humanes, S., Alegre, L.M., Castro, M., Rodriguez-Manas, L. \& Garcia-Garcia, F.J. (2015) Association of regional muscle strength with mortality and hospitalisation in older people. Age Ageing, 44, 790-795.

Guerra, R.S., Amaral, T.F., Sousa, A.S., Pichel, F., Restivo, M.T., Ferreira, S. \& Fonseca, I. (2015) Handgrip strength measurement as a predictor of hospitalization costs. Eur. J. Clin. Nutr., 69, 187-192.

Hamer, M., Batty, G.D. \& Kivimaki, M. (2015) Sarcopenic obesity and risk of new onset depressive symptoms in older adults: English Longitudinal Study of Ageing. Int. J. Obes. (Lond), 39, 1717-1720.

Jang, J.Y. \& Kim, J. (2015) Association between handgrip strength and cognitive impairment in elderly Koreans: a populationbased cross-sectional study. J. Phys. Ther. Sci., 27, 39113915.

Janssen, I., Heymsfield, S.B. \& Ross, R. (2002) Low relative skeletal muscle mass (sarcopenia) in older persons is associated with functional impairment and physical disability. J. Am. Geriatr. Soc., 50, 889-896.

Jeong, S. \& Jang, J.Y. (2017) Association between physical activity and cognitive dysfunction in the Korean: a crosssectional study. Exerc. Med., 1, 3.

Kim, J.M., Shin, I.S., Yoon, J.S. \& Lee, H.Y. (2003) Comparison of diagnostic validities between MMSE-K and K-MMSE for screening of dementia. J. Korean Neuropsychiatr. Asso., 42, 124-130.

Korea Employment Information Service (KEIS) (2015) Korean Longitudinal Study of Ageing.

http://survey.keis.or.kr/eng/klosa/klosa01.jsp

[Accessed: December 6, 2016].

Menant, J.C., Weber, F., Lo, J., Sturnieks, D.L., Close, J.C., Sachdev, P.S., Brodaty, H. \& Lord, S.R. (2017) Strength measures are better than muscle mass measures in predicting health-related outcomes in older people: time to abandon the term sarcopenia? Osteoporos. Int., 28, 59-70.

Mijnarends, D.M., Koster, A., Schols, J.M., Meijers, J.M., Halfens, R.J., Gudnason, V., Eiriksdottir, G., Siggeirsdottir, K., Sigurdsson, S., Jonsson, P.V., Meirelles, O. \& Harris, T. (2016) Physical activity and incidence of sarcopenia: the populationbased AGES-Reykjavik study. Age Ageing, 45, 614-620.

Mori, R., Shamoto, H., Maeda, K. \& Wakabayashi, H. (2016) Sarcopenia is a possible independent risk factor of cognitive decline in community-dwelling older people. J. Am. Med. Dir. Assoc., 17, 559-560.

Murad, K., Goff, D.C. Jr., Morgan, T.M., Burke, G.L., Bartz, T.M., Kizer, J.R., Chaudhry, S.I., Gottdiener, J.S. \& Kitzman, D.W. (2015) Burden of comorbidities and functional and cognitive impairments in elderly patients at the initial diagnosis of heart failure and their impact on total mortality: the Cardiovascular Health Study. JACC Heart Fail., 3, 542-550.

Nishiguchi, S., Yamada, M., Fukutani, N., Adachi, D., Tashiro, Y., Hotta, T., Morino, S., Shirooka, H., Nozaki, Y., Hirata, H., Yamaguchi, M., Arai, H., Tsuboyama, T. \& Aoyama, T. (2015) Differential association of frailty with cognitive decline and sarcopenia in community-dwelling older adults. J. Am. Med. Dir. Assoc., 16, 120-124.
Nourhashemi, F., Andrieu, S., Gillette-Guyonnet, S., Reynish, E., Albarede, J.L., Grandjean, H. \& Vellas, B. (2002) Is there a relationship between fat-free soft tissue mass and low cognitive function? Results from a study of 7,105 women. J. Am. Geriatr. Soc., 50, 1796-1801.

Pedersen, B.K. \& Febbraio, M.A. (2012) Muscles, exercise and obesity: skeletal muscle as a secretory organ. Nat. Rev. Endocrinol., 8, 457-465.

R Core Team (2017) R: A language and environment for statistical computing. https://www.R-project.org/ [Accessed: October 11, 2017].

Raji, M.A., Kuo, Y.F., Snih, S.A., Markides, K.S., Peek, M.K. \& Ottenbacher, K.J. (2005) Cognitive status, muscle strength, and subsequent disability in older Mexican Americans. J. Am. Geriatr. Soc., 53, 1462-1468.

Roberts, H.C., Denison, H.J., Martin, H.J., Patel, H.P., Syddall, H., Cooper, C. \& Sayer, A.A. (2011) A review of the measurement of grip strength in clinical and epidemiological studies: towards a standardised approach. Age Ageing, 40, 423-429.

Rosano, C., Simonsick, E.M., Harris, T.B., Kritchevsky, S.B., Brach, J., Visser, M., Yaffe, K. \& Newman, A.B. (2005) Association between physical and cognitive function in healthy elderly: the health, aging and body composition study. Neuroepidemiology, 24, 8-14.

Sallinen, J., Stenholm, S., Rantanen, T., Heliovaara, M., Sainio, P. \& Koskinen, S. (2010) Hand-grip strength cut points to screen older persons at risk for mobility limitation. J. Am. Geriatr. Soc., 58, 1721-1726.

Salthouse, T.A. (1996) The processing-speed theory of adult age differences in cognition. Psychol. Rev., 103, 403-428.

Salthouse, T.A., Hambrick, D.Z. \& McGuthry, K.E. (1998) Shared age-related influences on cognitive and noncognitive variables. Psychol. Aging, 13, 486-500.

Scazufca, M., Almeida, O.P., Vallada, H.P., Tasse, W.A. \& Menezes, P.R. (2009) Limitations of the Mini-Mental State Examination for screening dementia in a community with low socioeconomic status: results from the Sao Paulo Ageing \& Health Study. Eur. Arch. Psychiatry Clin. Neurosci., 259, 8-15.

Sternang, O., Reynolds, C.A., Finkel, D., Ernsth-Bravell, M., Pedersen, N.L. \& Dahl Aslan, A.K. (2016) Grip strength and cognitive abilities: associations in old age. J. Gerontol. B Psychol. Sci. Soc. Sci., 71, 841-848

The Ministry of Health and Welfare (2013) The Physical Activity Guide for Koreans.

http://health.mw.go.kr/ReferenceRoomArea/HealthFileRoom/ healthFileDetail.do?ED $\mathrm{NO}=1851$

[Accessed: December 6, 2016].

World Health Organization (2000) The Asia-Pacific perspective: redefining obesity and its treatment, Health Communications Australia, Sydney.

Xue, Q.L., Walston, J.D., Fried, L.P. \& Beamer, B.A. (2011) Prediction of risk of falling, physical disability, and frailty by rate of decline in grip strength: the women's health and aging study. Arch. Intern. Med., 171, 1119-1121.

Zhu, W., Wadley, V.G., Howard, V.J., Hutto, B., Blair, S.N. \& Hooker, S.P. (2017) Objectively measured physical activity and cognitive function in older adults. Med. Sci. Sports Exerc., 49, 47-53. 